\title{
Prolégomènes à la phraséologie comparée en langue de spécialité : exemple de l'anglais et du français de la finance
}

\section{Catherine Resche}

\section{(2) OpenEdition \\ Journals \\ Édition électronique \\ URL : http://journals.openedition.org/asp/3347 \\ DOI : 10.4000/asp.3347 \\ ISSN : 2108-6354 \\ Éditeur \\ Groupe d'étude et de recherche en anglais de spécialité}

\section{Édition imprimée}

Date de publication : 1 décembre 1997

Pagination : 487-503

ISSN : 1246-8185

\section{Référence électronique}

Catherine Resche, «Prolégomènes à la phraséologie comparée en langue de spécialité : exemple de l'anglais et du français de la finance », ASp [En ligne], 15-18 | 1997, mis en ligne le 04 mars 2013 , consulté le 30 avril 2019. URL : http://journals.openedition.org/asp/3347 ; DOI : 10.4000/asp.3347

Ce document a été généré automatiquement le 30 avril 2019

Tous droits réservés 


\title{
Prolégomènes à la phraséologie comparée en langue de spécialité : exemple de l'anglais et du français de la finance
}

\author{
Catherine Resche
}

1 En guise de déclaration préliminaire, j'aimerais préciser que je ne prétends pas me poser en pionnière puisque la phraséologie en langue générale d'abord, puis en langue de spécialité, fait l'objet d'une attention particulière depuis déjà quelques années. En revanche, au niveau bilingue, les travaux sont plus récents ; or, c'est précisément l'aspect comparatif (anglais-français) qui est au centre de mes préoccupations, puisque l'enseignante que je suis est sans cesse confrontée au problème d'aider au mieux les étudiants en économie à acquérir les outils linguistiques qui leur permettront de s'exprimer dans une langue aussi authentique que possible. Les quelques remarques que je propose ici sont donc le fruit d'une recherche contrastive que j'ai entreprise il y a plusieurs années par le biais de la terminologie comparée dans le domaine de la bourse au départ, puis élargi au domaine de la finance de manière plus générale. Elles sont le reflet d'une évolution logique et marquent une étape nouvelle dans mon approche de cette langue de spécialité.

2 Pour en venir maintenant au propos annoncé, il importe de souligner que si, par le passé, on a pu noter une fâcheuse tendance à réduire l'étude des langues étrangères et des langues de spécialité à des listes d'unités simples de la langue source accompagnées de leurs équivalents dans la langue cible (sous forme de glossaires, vocabulaires ou lexiques), depuis une décennie, une approche nouvelle s'est imposée. Avec l'unité terminologique, comme j'ai eu l'occasion de le souligner à plusieurs reprises par le passé $(1986,1995)$, ce sont également des unités plus complexes qui sont prises en compte. Il s'agit maintenant de franchir un pas supplémentaire avec l'approche phraséologique que je souhaite décrire ici. En vérité, l'attention s'est d'abord fixée sur l'unité la plus simple de la phrase, le mot, pour passer à l'unité terminologique puis maintenant à l'unité phraséologique, ce 
qui nous rapproche, nous le verrons, d'un retour à la phrase complète. II s'agit bien d'une évolution et non pas d'une révolution et mon but n'est évidemment pas de dénigrer les acquis antérieurs mais au contraire de construire à partir des bases établies. C'est pourquoi je propose dans un premier temps de faire ressortir les liens qui existent entre terminologie et phraséologie et de préciser les notions d'unité phraséologique et de cooccurrents. Il s'agira ensuite d'aborder l'aspect méthodologique et d'étudier la problématique à la lumière d'une recherche concentrée sur trois termes-noyaux. Enfin, j'évoquerai l'aspect plus pratique de la phraséographie en posant le problème de la mise en forme de fiches phraséo-terminologiques bilingues.

\section{Terminologie et phraséologie : complémentarité}

3 C'est après avoir lu Wuster pour qui la terminologie était un point de rencontre entre la logique, l'ontologie, la documentation et la linguistique que, dès 1983, la nécessité m'est apparue de donner à l'unité terminologique une acception beaucoup plus large que celle de l'époque, afin de tenir compte des semi-figements par exemple, et surtout pour mieux aider à replacer le terme dans son contexte discursif. Or, la fiche terminologique type, même avec ses multiples rubriques, ne permettait pas de rendre compte de manière satisfaisante des faisceaux de combinaisons des entrées. Il y avait donc lieu de chercher à remédier à cette imperfection. Mais déjà, la terminologie, en s'intéressant à la formulation de concepts analysés au préalable, ouvrait des horizons nouveaux en permettant de prendre en considération des entrées plus complexes. De la même façon qu'il a fallu définir la notion de " terme » et déterminer des critères de découpage pour l'unité terminologique (UT), il convient maintenant de s'entendre sur les notions d'unité phraséologique (UP) et de cooccurrents ; il est également indispensable de se pencher sur le problème du repérage des UP.

\subsection{Les unités phraséologiques}

Des définitions avancées par les uns et les autres, il ressort une constante, que l'on pourrait résumer de la manière suivante : ce sont des « combinaisons récurrentes, plus ou moins stabilisées, de formes lexicales et grammaticales » (Fiala $1987: 32$ ).

5 Ainsi exprimée, la notion laisse, on en convient, une grande latitude à l'interprétation. Pour mieux la cerner, on précisera que ces combinaisons sont composées « d'éléments linguistiques propres à un domaine de spécialité, dont l'un est un terme noyau, qui sont liés sémantiquement et syntaxiquement et pour lesquels il existe une contrainte paradigmatique » (Blais 1993: 52).

6 Le premier problème qui se pose est la sélection du terme noyau. C'est là, à mon sens, que la terminologie apporte une aide précieuse. En effet, puisque la délimitation de l'entrée terminologique a déjà donné lieu à une analyse conceptuelle, on peut très bien considérer que c'est à partir de l'UT que l'on va travailler et rechercher les combinaisons récurrentes. Le concept de terme-noyau est donc intéressant à double titre; d'une part, il symbolise le lien entre terminologie et phraséologie et d'autre part, il permet de répondre à un problème qu'on ne peut négliger, à savoir qu'il faut éviter de confondre UP et terme composé. Dès lors que l'on part de l'UT, ce problème n'en est plus un puisqu'on a appris à reconnaître l'unité composée comme une suite dans laquelle chaque élément de la composition contient un trait définitoire supplémentaire. Ainsi «demand-pull inflation, 
cost-push inflation, import-price-push inflation » sont des UT et non des UP. On pourrait donc avancer l'idée que l'UP a pour pivot l'UT. L'UP serait la somme des cooccurrents dont l'usage consacre l'association avec le terme-noyau, sachant que ce dernier peut lui-même être constitué de plusieurs éléments. On résumera l'UP par la formule suivante :

$\mathrm{UP}=\mathrm{UT}+$ cooccurrents

\subsection{Les cooccurrents}

7 Il s'agit maintenant de cerner la notion de cooccurrents, qu'il faut distinguer des collocateurs afin de ne pas retenir systématiquement tous les éléments qui encadrent, précèdent ou suivent le terme-noyau. Il faut cependant identifier les associations privilégiées par l'usage, qui ne sont pas pour autant figées, mais qui témoignent d'une certaine affinité. On parlera alors de figements à divers degrés, et c'est là que réside toute la difficulté car ces unités restent ouvertes à certaines variations. Les deux définitions déjà citées en référence pour cerner la notion d'UP soulignent la double préoccupation "lexicale et grammaticale" ou "sémantique et syntaxique» qui peut orienter le repérage des cooccurrents. L'emprunt de la LS à la langue commune peut en effet ou bien s'accompagner d'un détournement de sens ou bien conduire à une réécriture de la combinatoire grammaticale. Il semble évident que dans les deux cas, si l'on veut mieux rendre compte du discours, de la langue en fonctionnement et surtout dans l'optique de la phraséologie bilingue d'une LS, il faut souligner les écarts par rapport à la langue générale. Mais puisque le travail se fait sur deux langues, il faut concevoir que telle UP s'écartant de la «norme » du français général ne correspondra pas forcément à une UP de l'anglais de spécialité qui s'écarte elle aussi de l'anglais général. On entrevoit déjà la nécessité de rendre compte également des parallélismes, convergences et tournures équivalentes, ce qui me conduit à poser le problème de la longueur des UP et du type de cooccurrents à retenir.

\subsection{Longueur des UP}

8 Puisqu'on a établi un lien entre UT et UP, il va de soi que l'UP est automatiquement plus longue que l'UT qui lui sert de base. S'il y a consensus sur le segment minimum, il n'en va pas de même pour ce qui est de la limite supérieure. Certains pensent que l'UP n'atteint jamais la structure de la phrase (Blais 1993 : 53), d'autres assimilent l'UP à « toute entité digne d'intérêt et plus grande que l'unité terminologique standard» (Gouadec 1992 : 550), n'excluant donc pas d'aller jusqu'à la phrase si besoin est. Il est vrai que j'ai moi-même par le passé (1986) cité des exemples de tournures propres au domaine boursier qui étaient en fait des dictons ou préceptes et constituaient des phrases entières ; à mon avis, il faut en rendre compte dans l'optique de la phraséologie pour témoigner de leur existence et éviter des traductions approximatives. Je citerai pour mémoire :

Les arbres ne montent jamais jusqu'au ciel.

Trees don't grow to the sky.

Where there's a tip, there's a tap.

Your first loss is your best loss.

Il faut savoir sacrifier la main plutôt que perdre le bras.

9 Ces quelques exemples suffiront à corriger l'opinion avancée par certains selon laquelle « les LS ne comptent aucun proverbe ou dicton »! (Roberts $1993: 42$ ) 
10 Dans le même ordre d'idée, l'UP pourra s'allonger jusqu'à la phrase pour indiquer les métaphores récurrentes, surtout dans une optique bilingue. Il se trouve qu'en m'intéressant au domaine de la bourse puis à celui de la conjoncture économique pour cette étude précise, il m'est apparu qu'un nombre de champs métaphoriques semblait s'inscrire dans une certaine logique en s'appuyant sur des métaphores déjà lexicalisées qui reflétaient la façon d'appréhender la réalité et venaient en écho de l'expérience générale humaine révélatrice de telle ou telle civilisation. II y a donc lieu d'en témoigner, ne serait-ce que pour faire ressortir les points de convergence ou les dissemblances entre langue source et langue cible. Je reviendrai sur ce point un peu plus loin.

11 Si la phraséologie s'avère être complémentaire par rapport à la terminologie, c'est bien en donnant au traducteur, au technicien, au spécialiste de la LS, à l'enseignant, à l'étudiant, un maximum d'éléments pouvant les aider à saisir et rendre le sens de la langue en situation de manière encore plus authentique et compréhensible. Il est désormais clair pour tous que la terminologie est à la croisée des chemins entre langue et connaissance. Elle a d'ailleurs été décrite comme «l'alphabet de l'information » (Guyon 1995 : 21). Le rôle de la phraséologie est complémentaire en ce sens qu'elle aide à organiser cet alphabet dans la mesure où, en s'intéressant à la combinaison de chaque terme avec d'autres, dans le respect des règles de la syntaxe et de l'usage, elle permet de mieux construire l'information et d'assurer sa circulation et sa transmission.

\section{Méthodologie et problématique}

12 J'évoquerai maintenant les questions d'ordre méthodologique qui m'ont été inspirées par le travail de repérage et de sélection des UP auquel je me suis livrée, dans un contexte que j'ai résolument restreint à un sous-domaine, celui de la conjoncture économique, tant ma conviction est profonde qu'il est illusoire de parler de la langue économique dans son ensemble comme s'il s'agissait d'un tout homogène ; il m'apparaît beaucoup plus sage de procéder ainsi, dans la mesure où il $\mathrm{y}$ a une pluralité de technolectes à l'intérieur même de ce domaine.

\subsection{Corpus et cadre de l'étude}

13 Trois termes-noyaux ont servi de point d'ancrage à mon investigation. Ils présentent le double avantage d'être des notions incontournables de ce sous-domaine et d'être connus de tous : il s'agit de l'inflation/inflation, la croissance (économique)/(economic) growth et des taux d'intérêt/interest rates.

Quant au corpus, je l'ai délibérément tiré non pas d'ouvrages de grands économistes, mais de la presse spécialisée d'une part (The Financial Times, The Wall Street Journal, Business Week , The Economist, Le Journal des Finances, Investir, Le Revenu Français, Les Échos) et d'autre part de documents émanant des banques centrales (The Federal Reserve, The Bank of England et la Banque de France) ainsi que d'autres institutions bancaires (Crédit Agricole, Citibank). Pendant un an, j'ai consciencieusement dépouillé articles et documents portant sur les problèmes de croissance, inflation et taux d'intérêt. À partir de là, j'ai établi des listes et, après avoir confronté les différentes sources, je n'ai retenu que les UP qui étaient communes à la presse et aux documents des banques, ceci dans un souci de fiabilité, pour éviter les effets de style éventuels propres à tel ou tel journaliste. Ces listes sont 
reproduites en annexe, ordonnées en fonction de la place du terme-noyau par rapport aux cooccurrents et présentées par ordre alphabétique à l'intérieur de chaque groupe afin d'en faciliter la lecture.

Mon propos n'est pas de suggérer une typologie des UP à ce stade car une étude sur trois termes-noyaux peut très bien ne pas refléter l'éventail complet des combinatoires. Je dirai simplement qu'au vu des relevés effectués à cette occasion, il se trouve que les principaux cooccurrents permettraient la classification figurant dans le tableau 1.

Tableau 1

\begin{tabular}{|l|l|}
\hline \multicolumn{1}{|c|}{ Pour l'anglais } & \multicolumn{1}{|c|}{ Pour le français } \\
\hline UT + nom & UT + adj \\
UT-Adj & UT + prép + nom \\
Adj + UT & UT + prép + adj + nom \\
Adj-UT + nom & UT + adv + adj \\
Préfixe-UT + nom & Adj + UT \\
Nom + prép + UT & nom + prép +UT \\
UT + vbe & UT + vbe \\
Vbe + UT & vbe + UT \\
Vbe + prép + UT & vbe + prép + UT \\
Vbe + UT + post & \\
Vbe + UT + adj & \\
\hline
\end{tabular}

\subsection{Critères de sélection}

16 À la lecture des listes, on peut légitimement se poser la question des critères à adopter pour ne pas alourdir démesurément les données phraséologiques, au risque de constituer une gêne plutôt qu'une aide pour le lecteur. C'est pourquoi, sans prétendre apporter de réponses catégoriques, j'aimerais évoquer un certain nombre de problèmes et faire part de mes remarques.

\subsubsection{La fréquence}

17 Ce critère pourrait sembler aller de soi et constituer une garantie. En effet, la présence d'associations récurrentes dans les contextes émanant de sources diverses permet des recoupements qui laissent penser que, de toute évidence, on est en présence d'une UP digne d'intérêt. Faut-il pour autant considérer la fréquence comme une condition nécessaire et suffisante ? Ne risque-t-on pas dans ce cas ou bien de considérer comme UP une association qui, bien que récurrente, ne reflète qu'une mode passagère ou au contraire de laisser de côté une UP moins fréquente pour la simple raison que la conjoncture ne porte guère à l'utiliser, mais qui est tout aussi intéressante et révélatrice? 


\subsubsection{La prévisibilité}

18 J'entends par là soulever le problème de retournements prévisibles de catégories grammaticales du type nominalisation ou adjectivation. Si l'on considère les UP suivantes, relevées dans le cadre de cette étude :

quiescent inflation, inflation is quiescent, the quiescence of inflation

19 la tentation est forte de songer à alléger le dossier phraséologique en ne mentionnant qu'une de ces UP et en considérant que les autres seront acquises. La question à résoudre est de savoir si une majorité d'adjectifs utilisés comme attributs peuvent indifféremment être rencontrés comme épithètes dans le respect de la langue de spécialité sur laquelle on travaille; si tel est le cas, on pourrait effectivement se contenter de ne signaler que les exceptions, car le lecteur saurait qu'en l'absence de précisions, la formule suivante est applicable pour l'anglais :

$U T+b e+a d j=a d j+U T$

De la même manière, si l'on pouvait affirmer qu'à une UP de type "inflation is quiescent ", on peut avec certitude substituer une autre UP du type "the quiescence of inflation », on pourrait là encore faire l'économie de l'information puisqu'il serait acquis que :

UT + be + adj = nom + prép + UT

21 Encore faudrait-il que le lecteur soit certain de la préposition à employer! Du côté français, il en irait de même ; par exemple, à partir des UP suivantes

croissance ralentie, ralentissement de la croissance,

la croissance ralentit, ralentir la croissance

ne serait-on pas tenté de conclure:

$\mathrm{UT}+$ adj = nom + prép + (art) +UT = (art) + UT + vbe = vbe + (art) + UT

Il est vrai qu'on retrouve un certain nombre d'exemples dans les UP relevées en annexe qui pourraient faire croire à l'émergence de constantes. Je citerai en illustration pour le français : «maîtriser l'inflation/la maîtrise de l'inflation; lutter contre l'inflation/la lutte contre l'inflation». En anglais, d'autres retournements seraient prévisibles du type «to control inflation / inflation control » ou encore « to cut interest rates/a cut in interest rates/an interest rate cut ».

Or, premièrement, rien ne permet pour l'instant d'affirmer que ces possibilités de retournements sont opératoires dans la majorité des cas ; et même lorsqu'elles existent, elles peuvent conduire à un changement de sens. Il suffit de considérer l'UP " connaître une croissance de $\mathrm{x} \%$ » pour se rendre à l'évidence: même si le nom correspondant existe, cette UP ne peut se transformer en « connaissance d'une croissance ».

Deuxièmement, quand bien même on pourrait attester l'existence de constantes et ne signaler qu'une seule UP pour automatiquement sous-entendre les transformations possibles, encore faudrait-il savoir exactement à quel public on s'adresse. N'oublions pas que nous travaillons dans une optique bilingue; si la LS emprunte au registre de la langue générale, il faut donc s'assurer que les lecteurs auront une maitrise suffisante de celle-ci pour pouvoir concevoir et mettre en œuvre ces transpositions sans hésitation et ceci en français comme en anglais. 


\subsubsection{Connaissance du public cible}

Comme je viens de le souligner, bon nombre de choix peuvent être opérés à condition de garder à l'esprit les besoins et les aptitudes linguistiques du public auquel on s'adresse :

des francophones, des anglophones, des personnes plus ou moins familiarisées avec le domaine? Au moment de faire une traduction, est souvent préférable de voir confirmer une intuition que de courir risque d'employer une forme inhabituelle dans le registre de langue visé. (Humbley 1993 : 97)

Pour illustrer ce point, j'attirerai l'attention sur des problèmes qui me sont apparus au cours de cette étude.

\subsubsection{Le problème des prépositions : sens et choix}

Considérons le terme-noyau adjectif « inflationary » dans l'extrait de liste suivant :

« inflationary boom, - concerns, - consequences, - episodes, - fears, -spiral ».

S'il est vrai que l'on peut substituer « of inflation » à l'adjectif « inflationary » devant « spiral » ou « fears " (=a spiral of inflation, fears of inflation), « of » n'exprime pas pour autant le même rapport sémantique dans les deux cas. Par ailleurs, on ne pourra pas utiliser " of " avec «concerns». De plus, il faut que le lecteur sache comprendre «inflationary consequences " comme "inflation as a consequence » et non comme "the consequences of inflation ». Le choix des prépositions doit donc être signalé pour assurer un bon usage de la langue.

Une autre illustration viendra confirmer cette nécessité d'être précis dans les indications phraséologiques. On pourra comparer

to boost growth - a boost to growth

to cut interest rates - a cut in interest rates.

Les problèmes se posent également en français où, dans l'exemple suivant, deux verbes dont la construction est parallèle ne donnent pas naissance à des transformations en tous points semblables :

les taux d'intérêt se tendent - tension sur les taux d'intérêt

la courbe des taux d'intérêt s'est repentifiée - repentification de la courbe des taux d'intérêt

On pourrait aussi soulever le problème de la constante sémantique de prépositions telles que «sur » ou « de »; «sur » est loin de toujours indiquer le lieu et «de» n'atteste pas toujours un rapport d'appartenance...

\subsubsection{Le problème des articles}

La question des articles se pose notamment au niveau des UP de type «nom + de + UT » illustrées par «la robustesse de la croissance» ou «le rythme de la croissance». On peut légitimement se demander s'il faut, dans la liste des UP, mentionner à chaque fois l'article ou considérer que, de lui-même, le lecteur saura opérer le bon choix entre article indéfini, défini ou absence d'article. Effectivement, là encore, on pourrait envisager une certaine logique après avoir comparé les UP suivantes :

$\underline{\text { le }}$ rythme de la croissance/ $\underline{\text { un }}$ rythme de croissance

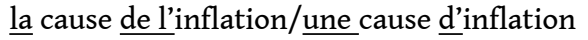



dans un certain registre pour exprimer, par exemple, les mouvements à la hausse ou à la baisse, ce qui ne manquera pas de donner lieu à de nombreuses répétitions. Pour ma part, je serais assez tentée de laisser de côté ces termes car « to rise, to increase, to decline, to drop, to go up/down... » se retrouveraient à coup sûr en cooccurrents de «shares, prices, inflation, growth, interest rates, unemployment... », pour ne citer que ces termes-noyaux. Si le public cible connaît tous les verbes ou noms qui décrivent ces fluctuations et les adverbes ou adjectifs qui peuvent les accompagner et les nuancer, est-il souhaitable de lui infliger cette surcharge d'informations? Ne risque-t-on pas l'asphyxie?

\subsubsection{Les UP de la langue générale}

De même, une bonne connaissance de la langue générale devrait permettre de déduire, à la lecture de l'UP «pressions inflationnistes " que des verbes tels que «subir» ou «faire face à, résister à, contenir ", peuvent s'associer à l'UP enregistrée, dans la mesure où ils sont des cooccurrents habituels de "pressions» dans l'usage courant. En anglais, " inflationary pressures » pourra naturellement être le sujet de «abate » ou le complément de « come under » ou " contain ». De la même manière, «policy » dans « inflationary policy" appellera naturellement «to run» ou « to implement », ce qui devrait permettre, selon le public auquel on s'adresse, de limiter le volume des UP. Mais, une nouvelle fois, la 
prudence s'impose, car il se peut que la LS fasse un usage de la langue générale qui lui est propre ; il appartiendra donc au phraséologue d'indiquer clairement cet usage.

\subsubsection{L'aspect métaphorique}

39

J'ai évoqué cet aspect qui me tient à cœur dans la première partie de cette étude et je souhaite m'y arrêter un instant pour souligner que même à partir d'un échantillon restreint, l'attention est attirée par des métaphores lexicalisées qui peuvent servir de clés aux champs métaphoriques prévisibles auxquels je faisais allusion. Je citerai quelques exemples, tirés des listes en annexe et dont il faut attester l'existence, soit pour montrer qu'on ne peut s'aventurer à traduire mot-à-mot, soit pour souligner la parfaite adéquation entre la langue source et la langue cible :

le moteur de la croissance/mettre un frein à la croissance

l'accélération de la croissance/relancer la croissance

la croissance ralentit/redémarre/s'accélère/s'asphyxie/repart

the engine of growth / a break to growth

to spark growth / to smother growth

growth accelerates / boils over / chugs along / chokes off / comes to a halt

En fonction de son degré de familiarisation avec la langue générale, le public pourra ainsi cerner les registres métaphoriques, les comparer d'une langue à l'autre et appréciera de voir son intuition confirmée en constatant l'existence d'autres métaphores du même registre, filées plus longuement et mentionnées comme UP. On le voit, de nombreuses questions restent à élucider. Des réponses ne pourront être apportées qu'après une étude d'une autre ampleur sur différentes LS. De ces réponses dépendront la forme et la norme de la fiche termino-phraséologique en LS bilingue pour laquelle j'aimerais maintenant ébaucher quelques hypothèses.

\section{Phraséographie}

41 Je n'entrerai pas dans le détail des considérations d'ordre technique concernant l'apport de l'informatique indéniablement indispensable pour un travail de dépistages des collocations à grande échelle.

42 Ce que je souhaite faire ici est de donner corps à un certain nombre d'idées sur la présentation des données phraséologiques. En fonction de ce que j'ai dit des liens entre terminologie et phraséologie, il me paraît souhaitable et logique de faire figurer sur une même fiche française, par exemple, les données terminologiques et phraséologiques, avec une simple indication de l'UT équivalente en anglais. Puis, en recherchant l'entrée anglaise ainsi indiquée, on pourrait consulter la fiche correspondante anglaise, conçue sur le même modèle. Le squelette d'une fiche pourrait se présenter comme au tableau 2.

Tableau 2. Fiche termino-phraséologique

\begin{tabular}{|l|l|}
\hline Entrée UT française & UT anglaise \\
\hline
\end{tabular}




\begin{tabular}{|l|l|}
\hline Données terminologiques & \\
Domaine / sous- domaine & \\
Informations grammaticales & \\
Définition & \\
Note supplémentaire & \\
Contexte & \\
Sigle & \\
Antonyme / Synonyme & \\
Variantes (orthographique, géographique, niveau de langue) & \\
Renvois (à d'autres UT du fichier) & \\
\hline Données phraséologiques & \\
UT + adjectif & \\
UT + prép + nom ........ (voir annexes) & \\
nom + prép + UT & \\
UT + vbe & \\
vbe + UT.....................(voir annexes) & \\
Proverbes / dictons & \\
Métaphores & \\
\hline
\end{tabular}

Peut-on envisager de faire cohabiter les deux fiches sur une fiche bilingue ? En dehors des problèmes de place et de lisibilité, on peut se demander si cela est souhaitable dans la mesure où les UP relevées dans une langue ne vont pas toutes systématiquement trouver leur correspondance dans l'autre langue. D'autre part, si l'on choisit d'ordonner les UP en tenant compte de l'agencement du terme-noyau par rapport aux cooccurrents, ne forcera-t-on pas l'utilisateur à passer en revue toutes les catégories d'UP recensées puisqu'on sait que les UP de la langue source ne verront pas leurs éléments ordonnancés de la même façon dans la langue cible?

Devrait-on adopter l'ordre alphabétique à l'intérieur des catégories d'UP ou réunir les expressions proches sur le plan sémantique? Devrait-on considérer que sur la fiche «taux d'intérêt », par exemple, on peut faire l'économie de l'UP «repentification de la courbe des taux d'intérêt » dans laquelle s'emboîtent deux UP, à savoir "repentification de la courbe » et "courbe des taux d'intérêt »? Dès lors que "courbe des taux d'intérêt » serait enregistrée, un simple signal codé de type astérisque $(*)$ pourrait permettre le renvoi à l'entrée « courbe » (domaine de l'analyse graphique), qui, elle, ferait mention de l'UP « repentification de la courbe ».

Je n'ai fait que survoler les questions car bien des pistes restent à explorer avant d'en arriver au stade de la mise en forme des fiches. Je me permettrai simplement d'ajouter que le choix de faire suivre les données terminologiques par les informations phraséologiques sur une même fiche ne doit pas donner lieu à un malentendu qui consisterait à considérer que la phraséologie est dépendante de la terminologie au sens de secondaire dans la hiérarchie. À mes yeux, si le lien entre les deux activités est évident, il est à concevoir en terme de complémentarité, dans un souci de plus grande exactitude dans l'approche d'une LS à caractère bilingue. En effet, l'identification de l'UT se situe au 
niveau notionnel, alors que l'approche phraséologique relève du fonctionnel. La phraséologie permet de mieux prendre en compte le tissu langagier, d'inventorier les trames spécifiques à la langue générale et a fortiori à la LS. L'intérêt de cette approche est évidemment de favoriser les comparaisons entre langue source et langue cible, d'améliorer la qualité et l'exactitude de l'expression lors du passage de l'une à l'autre.

\section{Conclusion}

Pour terminer, je souhaiterais revenir sur la notion de langue de spécialité en paraphrasant le mathématicien Henri Poincaré dans la description qu'il faisait de la science; mais là où il utilisait les termes «science » et "faits", je me permettrai de substituer les termes « langue » et « mots » pour rappeler :

on fait une (langue) avec des (mots) comme on fait une maison avec des pierres; mais une accumulation de (mots), n'est pas plus une (langue) qu'un tas de pierres n'est une maison.

Même si la phraséologie donne déjà une image plus fidèle, peut-on dire pour autant que les UP mises bout à bout suffisent à reproduire une langue de spécialité ? Il y a encore beaucoup d'interstices à sonder : les joints entre les pierres ont encore à livrer le secret de leur composition. Ceci veut dire qu'il faudrait se livrer à une étude méthodique de l'ensemble d'un domaine de spécialité, puis de l'ensemble d'une langue de spécialité, puis d'une multiplicité de LS, pour pouvoir enfin comparer les conclusions auxquelles de telles études permettraient d'aboutir avant de pouvoir se prononcer sur ce terme générique. Les questions sur lesquelles on doit s'interroger portent maintenant principalement sur la grammaire des LS: est-ce que, par exemple, telle catégorie grammaticale est plus densément représentée dans tel domaine du français de spécialité que dans le domaine équivalent en anglais ? La voix passive est-elle privilégiée dans telle LS en anglais plus que dans l'anglais général et plus que dans la LS française correspondante? Existe-t-il un parallélisme entre l'usage des déterminants ou des prépositions dans la LS et dans la langue générale? ...

Autant de questions, autant de pistes à défricher méthodiquement, par sous-domaine ou domaine... Vaste et ambitieux chantier qui s'ouvre ainsi devant nous tous qui portons un intérêt au sujet que celui de la description systématique d'une langue de spécialité dans une optique bilingue !

\section{BIBLIOGRAPHIE}

Blais, E. 1993. « Le phraséologisme. Une hypothèse de travail ». Terminologies nouvelles 10. RINT, $50-56$.

Fiala, P. 1987. «Pour une approche discursive de la phraséologie : remarques en vrac sur la locutionalité et quelques points de vue qui s'y rapportent, sans doute ». Langage et société 42. 
Gouadec, D. 1991. « Phraséologie et terminologie en traduction ». Actes du Colloque de Genève octobre 1991.

Guyon, A. 1995. « La terminologie au sein du ministère de l'Enseignement supérieur et de la recherche ». La terminologie en France. Hors-série $n^{\circ} 1$. Terminometro.

Humbley, J. 1993. «Exploitation d'un vocabulaire combinatoire : syntaxe, phraséologie, analyse conceptuelle ». Terminologies nouvelles 10. RINT, 95-102.

Resche, C. 1986. « La bourse des valeurs : terminologie comparée du français et de l'anglais ». Thèse de doctorat, Université de Rouen.

Resche, C. 1995. «La langue de la Bourse : convergences et divergences ». ASp 7-10, 91-112.

Roberts, R. 1993. « La phraséologie : état des connaissances ». Terminologies nouvelles 10. RINT, $36-42$.

\section{ANNEXES}

\section{ANNEXE 1 : unités phraséologiques anglaises}

\section{GROWTH}

$\underline{\mathrm{UT}}+$ nom : growth ceiling, - curve, - differential, - expectations, - factor, - forecast,- lag, models, - path, - policy, - prospects, - rate, - slump, - spurt, - surge, - target, - theory, trend, - year.

UT-adjectif : growth-led, growth-oriented.

Adjectif + UT : annualized growth, balanced -, buoyant -, continued -, continuing -, depressed -, excessive -, expected -, fast -, global -, healthy -, high -, hot -, improved -, inflationary -, lasting -, long-term -, moderate -, negative -, noninflationary -, planned -, rapid -, real -, renewed -, rising -, robust -, slackening -, slow -, sluggish -, solid -, spectacular -, steady -, strong -, sustainable -, sustained -, subdued -, subpar -, weak -, zero -

Adjectif-UT + nom : a high-growth period / policy, a slow-growth period.

Préfixe + UT + nom : pro-growth stance

Nom + prép + UT :

- In: an acceleration in growth, differentials in -, a pick-up/pickup in -, a revival in -, a slowdown in -, a speedup in -, an upswing in -, an upturn in -.

- Of: the engine of growth, the pattern of -, the rate of -.

- On : a brake on growth, a drag on -.

- To : a boost to growth.

UT + verbe : to accelerate, to boil over, to bounce back, to chug along, to come to a halt, to continue, to cool off, to decelerate, to decline, to dip, to fall back, to halt, to heat up, to lag, to persist, to pick up, to rebound, to resume, to slow (down), to surge, to surpass (forecasts), to warm up.

Verbe + UT : To absorb growth, to achieve -, to boost -, to brake -, to chill -, to compromise -, to cool -, to cramp -, to crush -, to deliver -, to enhance -, to fuel -, to gain -, to generate 
-, to hinder -, to moderate -, to post (strong) -, to promote -, to raise -, to reduce -, to restrain -, to sacrifice -, to slow -, to smother -, to spark -, to spur -, to squeeze -, to stimulate -, to sustain.

Verbe + prép + UT : to choke off -, to gnaw at -, to knock back -, to rein in -.

Verbe + UT + prép + nom : to bring growth to a standstill.

Verbe + UT + Adj : to push growth higher.

\section{INFLATION}

UT + nom : inflation accounting, - battle, - barometer, - bulge, - burst, - bout, - control, differentials, - dove, - expectations, - fears, - figures, - forecasts, - gap, - hawk, - indicator, - levels, - measures, - momentum, - outlook, - performance, - phobia, - premium, projection, - rate, - report, - risks, - target, - threats, - trend, - uptick.

UT-adjectif : inflation-adjusted ; --causing ; --fighting ; --free ; --indexed ; --induced ; -obsessed ; --phobic ; --prone ; --proof ; --ridden, --wary.

Adjectif (composé) + UT : accelerating inflation, anticipated -, built-in -, chronic -, continued -, core -, creeping -, current -, decelerating -, dormant -, double-digit -, escalating -, exported -, galoping -, global -, hidden -, imported -, mild -, moderate -, modest -, mounting -, ongoing -, open -, quiescent -, raging -, rampant -, roaring -, runaway -, self-feeding -, single-digit -, single-figure -, slow -, spiralling -, stable -, tame -, underlying -, unanticipated -, zero -.

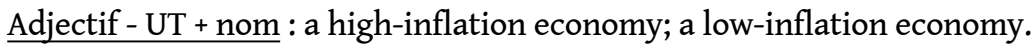

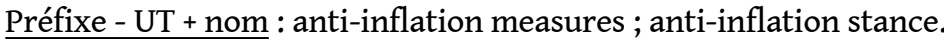

Nom + prép + UT :

- Against: the battle against inflation, hedges against -, a preemptive strike against -.

- For: the outlook for inflation, the target for - .

- of: a bout of -, a burst of -, a cooling of -, a downtrend of -, a flare-up of -, fears of -an outbreak of -, the pace of -, the quiescence of -, the rate of -, a reduction of -, a round of, the threat of -, an upswing of -, an uptick of -.

- On : a lid on inflation, (downward) pressure on -

- In: an acceleration in inflation, a deceleration in -, a decline in -, a downturn in -, an increase in -, a pick up/pickup in -, a reduction in -, a rise in -, an uptick in -, an upward tilt in - - To : a boost to inflation.

UT + verbe : to accelerate, to average..., to be at a low ebb, to be dead, to be in check, to be tame, to be well-contained, to bottom out, to come down, to cool, to creep up, to decelerate, to die, to doze, to escalate, to explode, to get out of control, to heat, to loom, to lurk, to moderate, to mount, to peak, to pick up (speed), to rebound, to run at (... \%), to shoot up, to slow down, to stay down, to speed up, to subside, to take off, to tick up.

Verbe + UT : to check -, to choke -, to combat -, to contain -, to control -, to curb -, to cut -, to decrease -, to fight -, to fuel -, to generate -, to hoist -, to ignite -, to moderate -, to reduce -, to rein in -, to repress -, to restrain -, to revive -, to slow -, to spark -, to squeeze -, to squelch -, to tame -, to tackle -, to trigger -, to overstate -, to yield. 
Verbe + prép + UT : to hedge against inflation, to adjust for inflation, to discount for inflation.

Verbe + UT + post : to bring - down, to force - down, to hold - down, to keep - at bay, to keep - down, to keep - in check, to keep - low, to push - up, to send - upwards, to wrestle down.

Phrase : All is quiet on the inflation front.

\section{INTEREST RATE(S)}

UT + nom : interest-rate bears, - boost, - ceiling, - changes, - decisions, - expectations, hike, - movements, - pressures, - risk, - runup, - spike, - swings, - volatility.

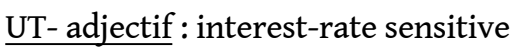

Adjectif + UT : domestic interest rates, falling -, floor -, global -, high -, key -, long-term -, low -, market -, nominal -, real -, rebounding -, rising -, short-term -, stable -, steady, volatile - .

Nom + prép + UT :

- In : changes in interest rates ; corrections in -, a cut in -, a deceleration in -, a dip in -, a downswing in -, a drop in -, a hike in -, a jump in -, a rebound in -, a retreat in -, a runup in -, shifts in -, a spike in -, swings in -, an uptick in -, volatility in -.

- Of: the capping of interest rates, the level of -, the seesaw movements of -, the upswing of - , the volatility of -.

UT + verbe : to behave, to be on a downward trend, to bottom out, to bounce around, to dip, to ease, to firm, to fluctuate, to follow an escalating course, to head up, to march up, to moderate, to move, to rebound, to retreat, to run higher than inflation, to shoot up, to soar, to take a dive, to trend down, to weaken.

Verbe + UT : To adjust interest rates, to affect -, to boost -, to cap -, to control -, to cut -, to ease -, to hike -, to hoist -, to lift -, to lower -, to manipulate -, to pare -, to raise -, to reduce -, to set -, to slash -, to steer -.

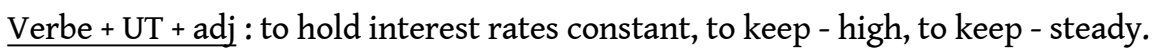

Verbe + prép + UT $:$ to decide on interest rates, to drive down -, to drive up -, to fiddle with -, to force down -, to force up -, to hold down -, to nudge up -, to push up -.

Verbe + nom + prép + UT : to keep control of interest rates, to put a cap on -, to resist a hike in -, to retain control of -.

\section{ANNEXE 2 : unités phraséologiques françaises}

\section{CROISSANCE}

UT + Adjectif : croissance annuelle, - anticipée, - durable, - fragile, - insuffisante, médiocre, - modérée, - non-inflationniste, - nulle, - ralentie, - réelle, - régulière, - saine, soutenue, - tendancielle.

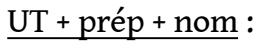

- $\underline{\text { à }: ~ c r o i s s a n c e ~ a ̀ ~ m o y e n ~ t e r m e, ~-~ a ̀ ~ l o n g ~ t e r m e ~}$

- en : croissance en baisse, - en hausse. 
$\underline{\mathrm{UT}+\text { art }+ \text { nom }}$ : croissance au plancher

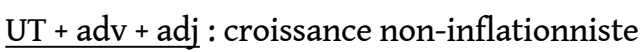

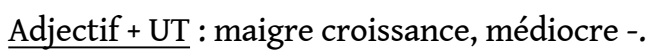

Nom + prép + UT :

\section{- à : frein à la croissance}

- de : accélération de la croissance, chiffres de la -, chute de la -, courbe de -, cycle de -, facteur de -, faiblesse de la -, fruits de la -, indicateur de -, modération de la -, moteur de la -, objectif de -, période de -, perspectives de -, phase de -, prévisions de -, ralentissement de la -, relance de la -, reprise de la -, ressort de la -, retombées de la -, retour de la -, robustesse de la -, rythme de -, surcroît de -, taux de -.

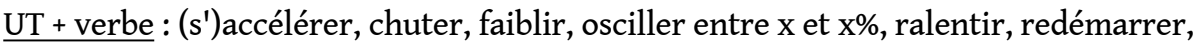
repartir, reprendre, revenir, s'améliorer, s'essouffler.

Verbe + UT : augmenter la croissance, atteindre une - de... \%, connaître une - de... \%, enregistrer une - de... \%, étouffer la -, freiner la -, gêner la -, gérer la -, limiter la -, prévoir une - de... \%, ralentir la -, recouvrer la -, relancer la -, répartir les fruits de la -, stimuler la -.

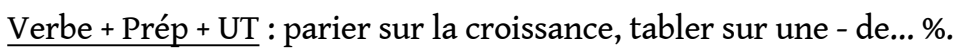

\section{INFLATION}

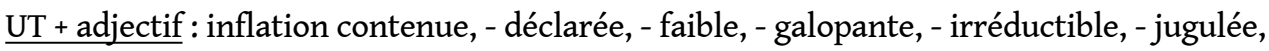
- larvée, - maîtrisée, - modérée, - nulle, - plate, - rampante, - réelle, - tendancielle, structurelle.

\section{$\underline{\mathrm{UT}}+$ prép + nom :}

- de : accès d'inflation, anticipation d'-, cause d'-, comptabilité d'-, dynamique de l'-, écart d'-, facteur d'-, flambée d'-, maitrise de l'-, niveau d'-, noyau dur de l'-, origine de l'-, partisan de l'-, période $\mathrm{d}^{\prime}-$, phénomène $\mathrm{d}^{\prime}$-, perspectives $\mathrm{d}^{\prime}$-, poussée $\mathrm{d}^{\prime}-$, ralentissement de l'-, ravages de l'-, recul de l'-, reprise de l'-, résurgence de l'-, retour de l'-, risque d'-, source d'-, taux d'-.

- contre : bataille contre l'inflation, lutte contre l' -, refuge contre l'-, remède contre l'-.

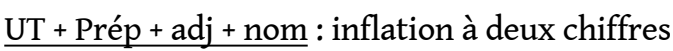

Loc prép + UT : à l'abri de l'inflation, en proie à l'-.

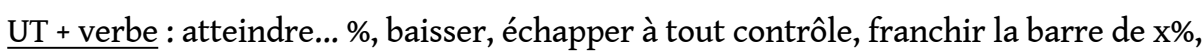
menacer, rester sous contrôle, s'amplifier, se maintenir (aux alentours de), s'aggraver, s'alimenter, se nourrir, se poursuivre.

Verbe + UT : aggraver l'-, alimenter l'-, coiffer l'-, combattre l'-, connaître une - de... \%, contenir l'-, contrôler l'-, enrayer l'-, entraîner l'-, étouffer l'-, étrangler l'-, freiner l'-, générer l'-, juguler l'-, limiter l'-, maîtriser l'-, nourrir l'-, réduire l'-, subir l'-, vaincre l'-. Verbe + prép + UT : faire échec à l'-, s'attaquer à l'-.

\section{TAUX D'INTERÊT}

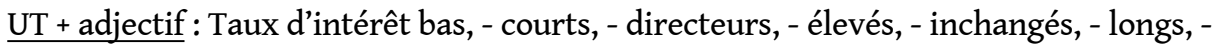
obligataires, - plafonds, - planchers, - réels, - réglementés, - révisables. 
$\underline{\mathrm{UT}+\text { prép }+(\text { adj })+\text { nom }}$ :

- à : taux d'intérêt à court terme, - à (dix) ans, - à long terme.

- en : taux d'intérêt en baisse, - en hausse.

Nom + prép + UT :

- $\underline{\text { des }}$ : augmentation des taux d'intérêt, baisse des -, barre des -, courbe des -, décrue des -, détente des -, évolution des -, fixation des -, fluctuations des -, hausse des -, maintien des -, maîtrise des -, modification des -, mouvements des -, niveau des -, plafond des -, réduction des -, relèvement des -, remontée des -, repli des -.

- entre : différentiels entre les -, écart entre les -.

- sur : action sur les-, tension sur les -.

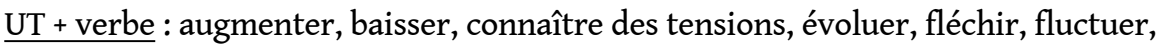
grimper, monter, rester au plancher, revenir, se détendre, se relâcher, se replier, se stabiliser, se tendre, tirer vers le bas, varier.

Verbe +UT : abaisser les -, administrer des -, augmenter les -, baisser les -, fixer les -, influencer les -, maintenir les -, maîtriser les -, pratiquer des -, réduire les -, relâcher les -, relever les -, servir des -.

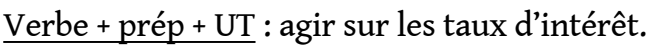

\section{RÉSUMÉS}

La phraséologie comparée est le prolongement logique et le complément de la terminologie comparée. Elle permet une approche plus complète de la réalité langagière de la langue de spécialité. L'unité phraséologique est constituée d'un terme-noyau et de cooccurrents dont la sélection est examinée ici à la lumière de différents critères: fréquence, prévisibilité, connaissance du public cible, de son niveau en langues et de ses besoins... Les questions soulevées sont étayées par l'étude des UP ayant pour termes-noyaux inflation, croissance, et taux d'intérêt dans les deux langues. Le problème pratique de la conception de la fiche phraséo-terminologique bilingue est envisagé. Si la phraséologie peut apporter une aide précieuse à l'étudiant et à l'enseignant d'une langue de spécialité, mais aussi au traducteur ou au spécialiste. En enrichissant l'analyse notionnelle effectuée dans une optique terminologique d'une dimension fonctionnelle illustrée par les unités phraséologiques répertoriées, elle ne permet pas encore de rendre compte de toute la complexité d'une langue de spécialité. Pour atteindre ce but, une description systématique de la syntaxe s'avère nécessaire.

Comparative phraseology can be seen as a logical evolution of and a complement to comparative terminology, offering a more accurate approach in the field of LSP and a more genuine reflection of discourse. In phraseology, the typical unit consists of a nucleus and collocates. The choice of the latter is examined in the light of such criteria as frequency, predictability and the target public's needs and degree of fluency... The questions that are examined here are based on a study focusing on the collocates of three terms, i.e., inflation, growth and interest rates in both French and English. The practical issue of how to manage to have terminological as well as phraseological data cohabit in a data bank is discussed. Though phraseology can undeniably provide students, teachers, translators and specialists with a helpful tool by adding a functional 
dimension to the notional approach of terminology, it still fails to fully account for all the intricacies of LSP. Only a methodical description of LSP syntax could help to reach this goal.

\section{INDEX}

Keywords : collocation, comparative phraseology, comparative terminology, LSP, nucleus, phraseography

Mots-clés : cooccurrent, langue de spécialité, phraséographie, phraséologie comparée, termenoyau, terminologie comparée

\section{AUTEUR}

\section{CATHERINE RESCHE}

Après l'agrégation d'anglais, Catherine Resche a choisi de faire un DEA de linguistique avant d'entreprendre une thèse de terminologie comparée sur la bourse de valeurs. Elle est maître de conférences à l'Université Paris2 Panthéon-Assas plus particulièrement chargée de l'enseignement de l'anglais de spécialité en DEA Monnaie-Finance-Banque. Elle fait également partie du Centre de terminologie économique et juridique de l'Université de Paris 1 . Elle s'intéresse à tous les aspects de la langue de spécialité : terminologie, phraséologie, dictons, métaphores, néologie, normalisation, siglaison et aux problèmes de la traduction spécialisée, de la création de banques de données et de dictionnaires informatisés. catherineresche@clubinternet.fr 